\title{
Optimal Sizing of RF Integrated Inductors for Power Transfer of Implantable Biosensors ${ }^{\dagger}$
}

\author{
Issa Sabiri 1,*, Hamid Bouyghf ${ }^{2}$ and Abdelhadi Raihani ${ }^{3}$ \\ 1 LSSDIA, ENSET Mohammedia, University of Hassan II Casablanca, Mohammedia, Morocco; \\ issa.sabiri@etu.fstm.ac.ma \\ 2 EEA \& TI Laboratory, Faculty of Science and Technology, University of Hassan II Casablanca, \\ Mohammedia, Morroco; hamid.bouyghf@gmail.com \\ 3 LSSDIA, ENSET Mohammedia, University of Hassan II Casablanca, Mohammedia, Morocco; \\ abraihani@yahoo.fr \\ * Correspondence: issa.sabiri@etu.fstm.ac.ma; Tel.: +212-610-60-6869 \\ + Presented at the 1st International Electronic Conference on Biosensors, 2-17 November 2020; Available \\ online: https://iecb2020.sciforum.net/.
}

Received: date; Accepted: date; Published: date

\begin{abstract}
Energy recovery methods are currently receiving a very great deal of attention from the research community. Especially, in the case of implantable biosensors where wireless energy transfer has become the main technique in these applications. An implant is a medical device manufactured to replace a missing biological structure, support a damaged biological structure, or enhance an existing biological structure. Biosensors are man-made devices, in contrast to a transplant, which is a transplanted biomedical tissue. The method of energy transfer eliminates the risk of skin infection, as well as the need for invasive surgery to change the battery. In this paper, we present the efficient approach to design an optimized octagonal spiral inductor operating at a frequency of $2.4 \mathrm{GHz}$ with an inductance $\mathrm{L}$ value of $4 \mathrm{nH}$ and a maximum factor of quality-Q. The principle part of this work is based on the use of a collection of methods called metaheuristics, which are approaches used to solve a wide range of optimization problems, in order to achieve a highperformance optimized design. The problem is represented by an objective function that will be implemented using MATLAB script and then the validation of the results obtained will be performed using the ADS microwave circuit simulation software.
\end{abstract}

Keywords: biosensors; wireless energy transfer; octagonal spiral inductor; metaheuristics; factor of quality Q

\section{Introduction}

Nowadays, a lot of research is focused on the realization of integrated inductors to meet the needs and more operational for various applications: in signal processing to enter the realization of low-noise amplifiers [1], in biomedical engineering [2] including applications related to energy storage [3]. The design of such components requires specific techniques and mainly the use of three types of materials: a conductive material, a magnetic material, which in most cases is ferrite, ironsilicon alloys or nickel-silicon alloys in the case of inductance with a core and sometimes an insulator. The performance of the component depends on several factors, on the one hand on the magnetic properties of the material [4], if it is a low-frequency application we generally find magnetic conductive materials with high permeability.

In this paper the objective will be the design and optimization of an integrated inductor in CMOS technology for biosensors using the Differential Evolution algorithm (DE). The study consists in using the differential evolution algorithm to design a planar integrated inductor of the octagonal spiral type 
operating at a frequency around $2.4 \mathrm{GHz}$ with a maximum quality factor and an inductance $\mathrm{L}$ which tends towards $4 \mathrm{nH}$, taking into account the different electrical and magnetic phenomena (the skin effect, the proximity effect...), which are reproduced in such structures.

\section{Materials and Methods}

\subsection{Physical Model of Spiral Inductor}

\subsubsection{Equivalent Model}

We have considered the equivalent circuit shown in Figure 1 to calculate the inductance value by the following equation:

$$
L_{S}=-\frac{1}{2 \pi f} \operatorname{Im}\left(\frac{1}{Y_{12}}\right)
$$

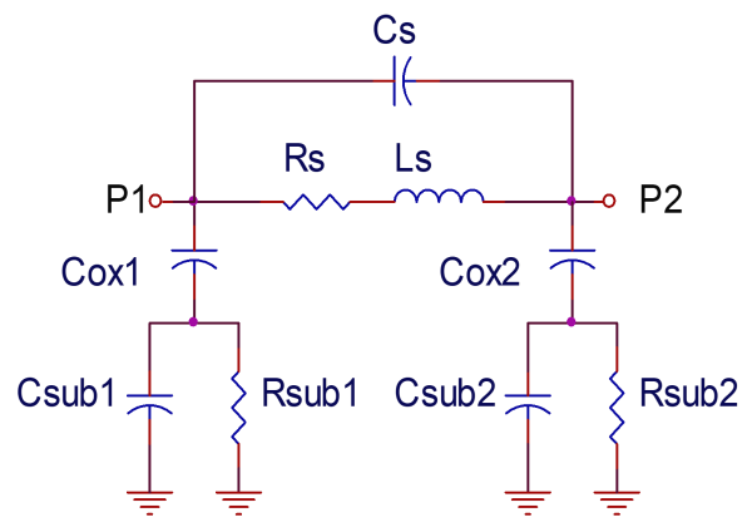

(a)

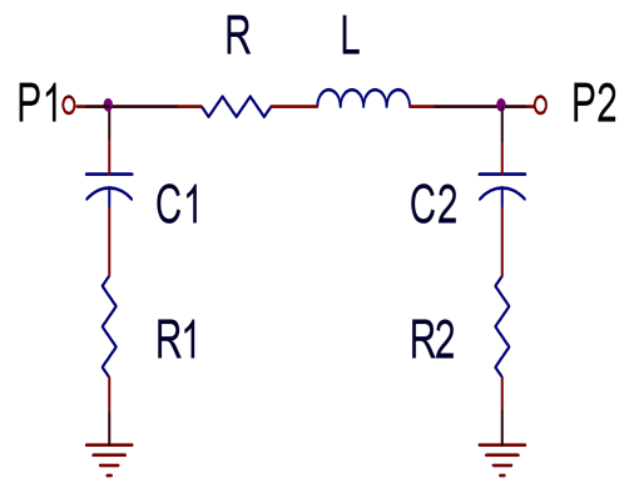

(b)

Figure 1. (a) physical model of octagonal inductor; (b) Simplified equivalent circuit.

The parameters of the physical model are calculated according to the geometrical parameters of the inductance by the following equations [5]:

- coupling capacity between the coils:

$$
C_{s}=n \cdot w^{2} \cdot \frac{\varepsilon_{o x}}{t_{o x M 1-M 2}}
$$

- Parasitic capacitance:

$$
C_{o x}=\frac{1}{2} \cdot l \cdot w \cdot \frac{\varepsilon_{o x}}{t_{o x}}
$$

- capacity of substrates:

$$
C_{s i}=\frac{1}{2} \cdot l \cdot w \cdot C_{s u b}
$$

- $\quad$ Resistance of the substrate:

$$
R_{s i}=\frac{2}{l \cdot w \cdot G_{\text {sub }}}
$$




\subsubsection{Inductance $\mathrm{Ls}$}

Several methods are presented in the literature to calculate the Ls such as wheeler's method [6], Baryan's method [7], Terman's method, monomial method and others. In our simulation we will use the monomial method because it remains valid for a small number of turns. The monomial expression used to calculate the self inductance is given by the following relation:

$$
L s=\beta \cdot D_{\text {out }}^{\alpha_{1}} \cdot W^{\alpha_{2}} \cdot D_{\text {avg }}^{\alpha_{3}} \cdot N^{\alpha_{4}} \cdot S^{\alpha_{5}}
$$

$N$ is the number of turns, $\beta, \alpha 1, \alpha 2, \alpha 3, \alpha 4$ and $\alpha 5$ are coefficients according to the geometrical shape of the inductance studied, $D_{\text {avg }}$ is calculated from inner diameter $D_{\text {in }}$ of the inductance outer diameter Dout by the following relation:

$$
\begin{gathered}
D_{\text {avg }}=\frac{\left(D_{\text {in }}+D_{\text {out }}\right)}{2} \\
D_{\text {out }}=D_{\text {in }}+2 \cdot N \cdot W+2 .(N-1) \cdot S
\end{gathered}
$$

The coefficients $\beta, \alpha 1, \alpha 2, \alpha 3, \alpha 4$ and $\alpha 5$ are given as follows in the Table 1 :

Table 1. Table of coefficients used in the monomial expression.

\begin{tabular}{ccccccc}
\hline Geometry & $\boldsymbol{\beta}$ & $\boldsymbol{\alpha} \mathbf{1}$ & $\boldsymbol{\alpha} \mathbf{2}$ & $\boldsymbol{\alpha} \mathbf{3}$ & $\boldsymbol{\alpha} \mathbf{4}$ & $\boldsymbol{\alpha} \mathbf{5}$ \\
\hline Octagonal & $1.33 \times 10^{-3}$ & -1.21 & -0.163 & 2.43 & 1.75 & -0.049 \\
\hline
\end{tabular}

\subsubsection{Factor of Quality-Q}

To calculate the quality factor $Q$, a parallel equivalent schema of the spiral inductance is proposed, this scheme is characterized by the parallel capacitance $\mathrm{Cp}$ and parallel resistance $\mathrm{Rp}$. The Figure 2 below shows the proposed parallel model.

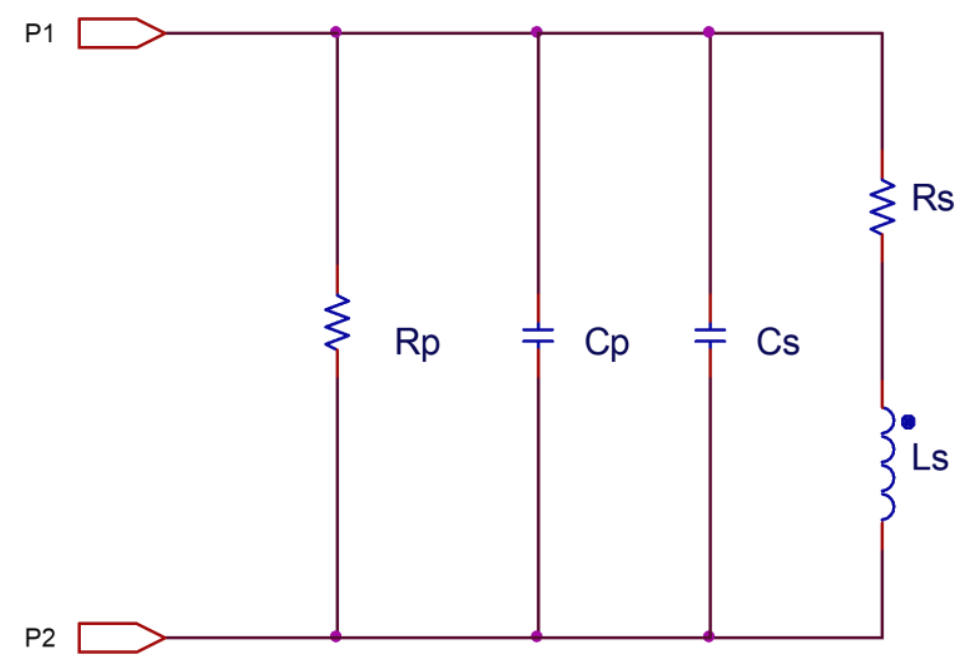

Figure 2. Parallel model of spiral inductance.

The quality factor is defined in the following equation as follows:

$$
Q=\frac{\omega L_{S}}{R_{S}} \cdot \frac{R_{P}}{R_{P}+\left[\left(\omega L_{S} / R_{S}\right)^{2}+1\right] R_{S}} \cdot\left[1-\frac{\left(C_{P}+C_{S}\right) R_{S}{ }^{2}}{L_{S}}-\omega^{2} L_{S}\left(C_{P}+C_{S}\right)\right]
$$




\subsection{Differential Evolutionary Algorithm (DE)}

Differential evolutions, or DE, has been proposed by Storn and Kenneth Price [8]. It is an algorithm inspired by genetics and evolutionary strategy, it is also based on the three operations: mutation, crossover and selection. The mutation generates new elements from certain elements randomly chooses then the crossing between the parents and the created elements and a selection operation to have only the most suitable elements in the generation.

\section{Objective Function}

We are looking to maximize the quality factor $\mathrm{Q}$ expressed in equation $\mathrm{x}$. This equation will be considered as a objective function $(\mathrm{OF})$, this amount to:

- Maximize the quality factor $\mathrm{Q}$ which depends on the geometrical parameters of the inductor $(\mathrm{N}, \mathrm{W}, \mathrm{S})$

- We will introduce $\mathrm{Qreq}=1 / \mathrm{Q}$, it represents the desired quality factor, so the problem is transformed towards a minimization of Qreq, in order to maximize the quality factor Q.

- All this will be done by the following constraints that must be satisfied:

$$
\begin{gathered}
2 \leq \mathrm{N} \leq 10 \\
20 \leq \mathrm{W} \leq 10 \\
3 \leq \mathrm{S} \leq 50
\end{gathered}
$$

\section{Results}

At 1000 iterations, after satisfying the stopping condition the algorithm provides the best combination of geometrical parameters corresponding to the optimal quality factor $Q$, the Figure 3 shows the result of the inductance optimization by the DE method. Figure 4 shows the MomentumADS simulation results $Q$ using the obtained optimal values by DE based method

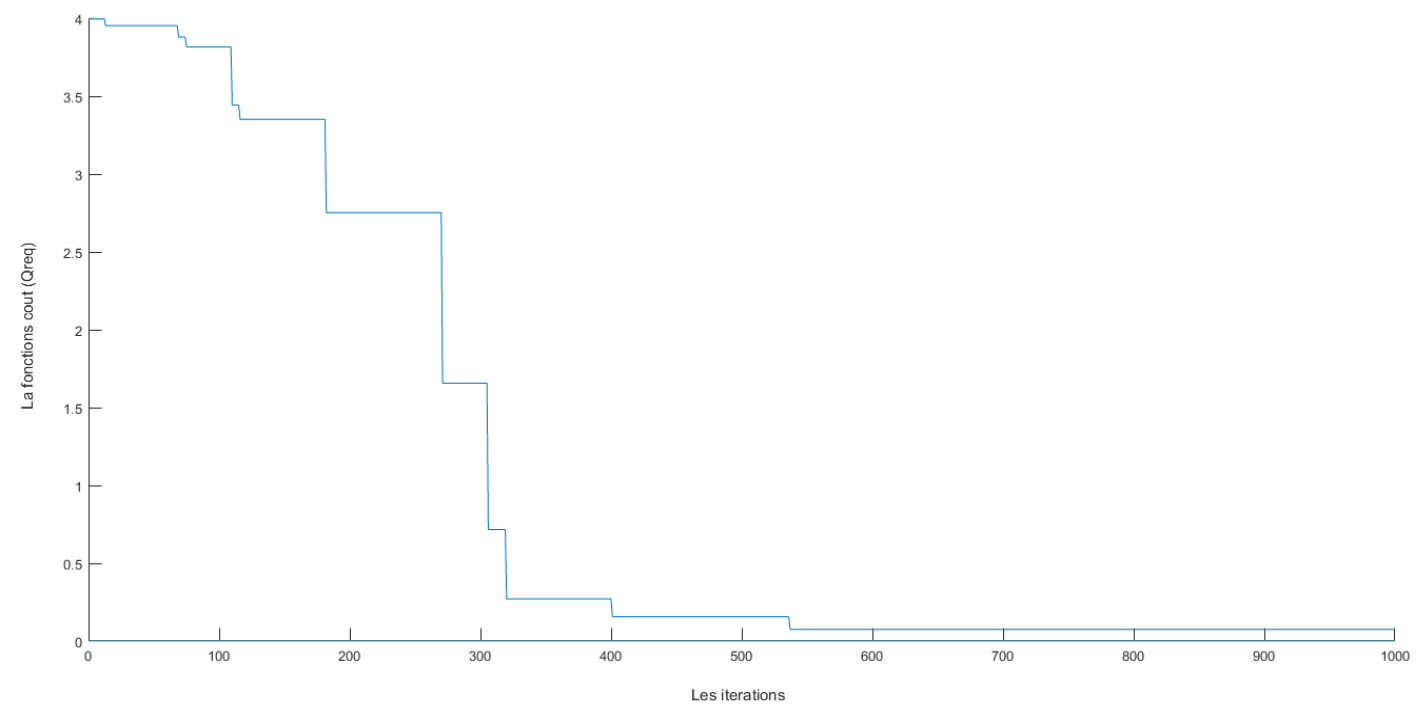

Figure 3. Objective function of the spiral inductor versus Iterations.

The Table 2 shows the optimal parameters given by the DE algorithm

Table 2. Parameters given by the DE algorithm.

\begin{tabular}{ccccccc}
\hline $\mathbf{S}(\mu \mathrm{m})$ & $\mathbf{w}(\mu \mathrm{m})$ & $\mathbf{N}$ & $\mathbf{D}_{\text {out }}(\mu \mathrm{m})$ & $\mathbf{L s r e q}_{(\mathbf{n H})}$ & $\mathbf{L D E}$ & $\mathbf{Q}_{\mathrm{DE}}$ \\
\hline 4.56 & 13.62 & 3.21 & 223 & 4 & 3.95 & 13.46 \\
\hline
\end{tabular}




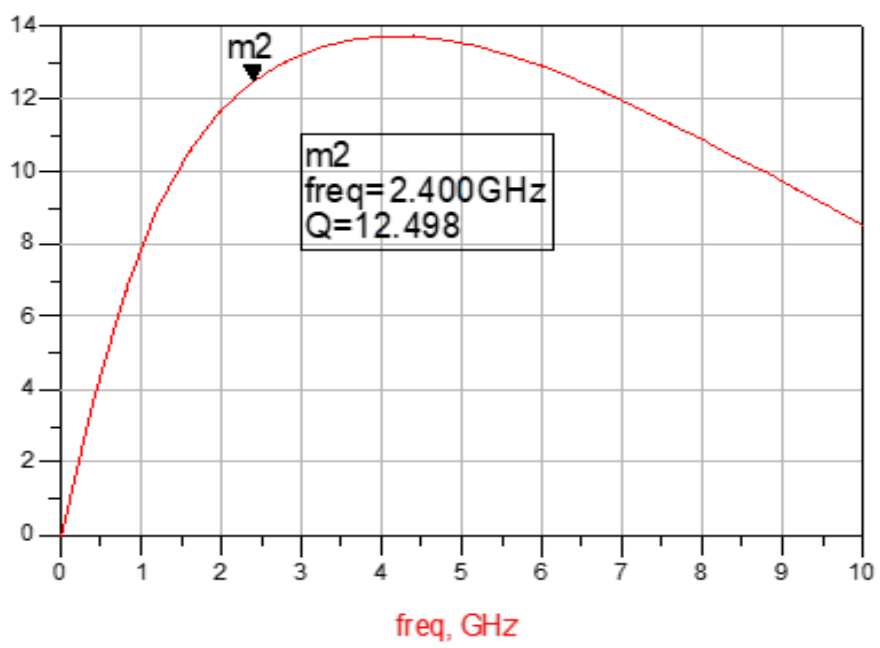

Figure 4. Simulation of quality factor $\mathrm{Q}$.

\section{Discussion}

The simulation has shown very clearly that an octagonal inductance can be designed with the desired criteria with a very high percentage, in fact we have obtained a fairly high-quality factor, and the simulation value is close to the value provided by the $\mathrm{DE}$ algorithm, with an error that does not exceed $7 \%$.

\section{References}

1. Jeamsaksiri, W.; Mercha, A.; Ramos, J.; Linten, D.; Thijs, S.; Jenei, S.; Detcheverry, C.; Wambacq, P.; Velghe, R.; Decoutere, S. Integration of a $90 \mathrm{~nm}$ RF CMOS technology (200 GHz fmax $-150 \mathrm{GHz}$ fT NMOS) demonstrated on a $5 \mathrm{GHz}$ LNA. In Proceedings of the Digest of Technical Papers. 2004 Symposium on VLSI Technology, Honolulu, HI, USA, USA, 15-17 June 2004; pp. 100-101.

2. Chen, L.Y.; Benjamin, C.K.T.; Alex, L.C.; Gregor, S.; Victor, T.; Darren, J.L.; Philip, H.S.W.; Michael, V.M.; $\mathrm{Bao}, \mathrm{Z}$. Continuous wireless pressure monitoring and mapping with ultra-small passive sensors for health monitoring and critical care. Nat. Commun. 2014, 5, 1-10.

3. Eom, K.; Jeong, J.; Lee, T.H.; Lee, S.E.; Jun, S.B.; Kim, S.J. Columnar transmitter based wireless power delivery system for implantable device in freely moving animals. In Proceedings of the Engineering in Medicine and Biology Society (EMBC), 2013 35th Annual International Conference of the IEEE, Osaka, Japan, 3-7 July 2013; pp. 1859-1862.

4. Yue, C.P.; Ryu, C.; Lau, J.; Lee, T.H.; Wong, S.S. A Physical model for planar spiral inductors on silicon. In Proceedings of the IEEE International Electron Devices Meeting (IEDM) Technical Digest, San Francisco, CA, USA, 8-11 December 1996; pp. 155-158.

5. Bouyghf, H.; Benhala, B.; Raihani, A. Analysis of the impact of metal thickness and geometric parameters on the quality factor-Q in integrated spiral inductors by means of artificial bee colony technique. Int. J. Electr. Comput. Eng. 2019, 9, 2918-2931.

6. Mohan, S.S.; Hershenson, M.d.; Boyd, S.P.; Lee, T.H. Simple accurate expressions for planar spiral inductances. IEEE J. Solid State

7. Bryan, H.E. Printed inductors and capacitors. Tele-Tech Electron. Ind. 1955, 14, 68

8. Storn, R.; Price, K. Differential evolution-A simple and efficient heuristic for global optimization over continuous spaces. J. Glob. Optim. 1997, 11, 341-359.

Publisher's Note: MDPI stays neutral with regard to jurisdictional claims in published maps and institutional affiliations.

(C) 2020 by the authors. Submitted for possible open access publication under the terms and conditions of the Creative Commons Attribution (CC BY) license (http://creativecommons.org/licenses/by/4.0/). 